\title{
Editorials
}

\section{Maternal mortality from eclampsia in developing countries: some progress, but still a major challenge}

Dan Benhamou MD

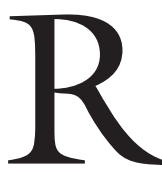

EADERS of the Journal will probably be surprised by the contents of the article by Brouh et al. ${ }^{1}$ published in this issue, which describes maternal mortality and intensive care unit (ICU) events of pregnant patients in Western Africa. Most readers are probably much more familiar with reports on maternal mortality from Western countries, including the Confidential Enquiries on maternal deaths in the United Kingdom (UK), ${ }^{2}$ the French Report on Maternal deaths, ${ }^{3}$ or descriptions of maternal deaths in the United States. ${ }^{4}$ These reports generally describe relatively small numbers of cases, usually over three-year periods, to generate sufficient sample sizes in order to satisfy the requirements of epidemiologic analyses. Interestingly, the UK Confidential Enquiry, ${ }^{2}$ which is now in its seventh iteration, emphasizes remarkable changes in the epidemiology of maternal deaths in the UK: a changing pattern in the factors associated with maternal deaths is becoming apparent, with an increasing role of obesity, diabetes, tobacco use, and psychiatric illness. This probably reflects what is happening, to varying degrees, in most other Western countries. These associated factors are now leading to deaths from causes almost unknown until recently, in patients from this relatively young age group. The increasing number of deaths from non-pregnancy related conditions (indirect causes) contrasts with a decreasing number of deaths from diseases associated with pregnancy, such as hemorrhage, preeclampsia, and obstructed labour (direct causes). Anesthesia is another cause of maternal mortality, for which a major improvement has been observed with about only one anesthesia-related death occurring per year in countries such as France or the UK. ${ }^{2,3}$ Improve- ments in organization of obstetrical services, development of strategies focusing on prevention of the major causes of death, and continuing medical education initiatives have been key factors in reducing the number of maternal deaths from direct causes. These positive changes have evolved, in part, because of the relatively high gross domestic product (GDP) in these industrialized countries, and because the proportion of GDP expenditures apportioned to health services is sufficiently large to facilitate technical and organizational changes in the delivery of health care services. The limits of these changes seem to have been reached, as maternal deaths observed in developed countries now tend to occur more often in poor migrants and/or socially vulnerable patients with a low income, for whom barriers exist in accessing the well organized general health services provided. ${ }^{5}$ Surprisingly, direct causes of maternal deaths in these patients are dissimilar to what has been observed previously in Western countries. Paradoxically, physicians and health care workers managing obstetric patients are discovering health problems that are more prevalent in lower socioeconomic classes, which represent the downside of an economically rich society.

The good news, however, is that preventable causes of maternal mortality have been declining in developed countries, and there is potential for improvement in several other parts of the world. The incidence of maternal deaths in developed countries is $50-100$ times lower than that observed today in Sub-Saharan Africa or South Asia, for example. ${ }^{6}$ In these countries, the leading causes of maternal death remain those that occur during labour and the immediate postpartum period, and these events are generally well controlled

CAN J ANESTH 2008/ 55: 7 / pp 397-402

From the Département d'Anesthésie-Réanimation, Hôpital de Bicêtre, France.

Address correspondence to: Pr Dan Benhamou, Département d'Anesthésie-Réanimation, Hôpital de Bicêtre, 78 rue du Général Leclerc, 94275 Le Kremlin Bicêtre Cedex, France. Phone: 33-1-45-21-34-41; Fax: 33-1-45-21-28-75; E-mail address: dan.benhamou@bct.aphp.fr 
in developed nations. Due to under-resourced health systems, maternal deaths continue to occur at a high rate, resulting primarily from direct causes. Obstetrical hemorrhage represents $30 \%$ of all causes of maternal death, and hypertensive crises are the origin of another $15 \% .^{7}$ When including sepsis, these three factors contribute to more than $50 \%$ of all maternal deaths in subSaharan Africa. ${ }^{6}$ Interestingly, these numbers are more favourable in certain countries. ${ }^{6}$ Because early detection of obstetrical disease, such as preeclampsia, is recognized to be of major clinical importance, focused initiatives have facilitated the deployment of skilled birth attendants and midwives to isolated settings and small villages to detect complications at an early stage. ${ }^{8}$ The importance of early detection is especially true for preeclampsia, which can be diagnosed with simple and easily available measures (regular blood pressure measurement, and urinalysis to monitor for proteinuria).

Maternal deaths now occur more often in hospitals ${ }^{6}$ suggesting that a larger number of parturients can have access to organized care, and are not limited to local care by family or proxies who have no formal medical training, and practice only traditional African medicine. A more recent development in some countries is that obstetric patients can be admitted to ICUs. This level of care is now available in the capital of Côte d'Ivoire, Abidjan, as described by Brouh et al. ${ }^{1}$ Although Côte d'Ivoire is considered to be part of Sub-Saharan Africa, it is located in its western part, and has a health care coverage which is slightly better that most of its neighbouring countries. Coccody and Yopougon are, however, two of only three university teaching hospitals in that country, reminding us of the limited access to health care in Western Africa, and especially in Côte d'Ivoire, where maternal mortality rates remain amongst the highest in the world $(>600$ per 100,000 live births). ${ }^{6}$

The data presented in the paper by Brouh et al. ${ }^{1}$ may leave us with mixed feelings. On one hand, some features of hospital care seem very similar to what can be seen in more developed countries, such as a high number of Cesarean deliveries, large numbers of patients receiving mechanical ventilation, and ready availability of low molecular weight heparins. On the other hand, the number of eclamptic crises in these two hospitals was much greater than most anesthesiologists would ever witness during their professional careers. Moreover, the large number of reported cases probably underestimates the real number of eclamptic seizures which had occurred in this setting during the study period. It appears likely that some patients had not been admitted to either of the two ICUs because of limited referral to emergency care centres. ${ }^{9}$ Furthermore, the availability of modern drugs and medical equipment in these two units should not cause one to overlook the situation in most other units. A survey in Uganda showed that only 6\% of anesthesiologists had the facility to deliver safe anesthesia for Cesarean delivery. ${ }^{10}$

Maternal mortality following eclamptic seizures was high in these two university teaching hospitals. Indeed, in most other trials, maternal mortality associated with eclampsia was much lower than the $16 \%$ incidence reported here. ${ }^{1}$ In the Collaborative Eclampsia Trial which evaluated women who had already had eclamptic seizures (secondary prevention), mortality was $5.1 \%$ in the control groups (receiving either diazepam or phenytoin). ${ }^{11}$ Women who had received magnesium sulfate $\left(\mathrm{MgSO}_{4}\right)$ had a $26 \%$ lower absolute risk of death, i.e., $3.8 \%$, but the difference was not statistically significant. In another trial, performed in India (before and after study), ${ }^{12}$ maternal mortality from eclampsia was significantly lower after $\mathrm{MgSO}_{4}$ had been introduced (4\%). Deaths were secondary to cerebrovascular hemorrhage, recurrent convulsions, or associated complications such as acute renal failure, jaundice, aspiration pneumonia and pulmonary edema. In trials in which $\mathrm{MgSO}_{4}$ was administered to severely preeclamptic patients who had not yet experienced seizures (primary prevention), maternal mortality was even lower $(0.4 \%$ incidence in the Magpie trial). ${ }^{13}$ In other words, the severity of the underlying disease and the failure to use $\mathrm{MgSO}_{4}$ were the major factors associated with an increased risk of death. As described by the authors, $\mathrm{MgSO}_{4}$ was not used in their unit at the time of the study, and diazepam, although less efficacious to prevent recurrence of seizures, was the drug most often used. This situation is not unique. Country-specific barriers, and potential facilitating factors relating to the drug's availability and use have been recently described. ${ }^{14}$ Most low-resource countries do not have guidelines mandating the use of $\mathrm{MgSO}_{4}$. There are also financial, logistic, and safety concerns associated with the universal administration of $\mathrm{MgSO}_{4}$. Many institutions in the developing world lack the necessary equipment and expertise to administer the medication, and many preeclamptic patients with imminent eclampsia thus do not receive $\mathrm{MgSO}_{4}$ before their first seizure. Morbidity and mortality arising from $\mathrm{MgSO}_{4}$ administration errors and toxicity have been widely publicized, but most health care workers have not been trained to administer the drug, and their confidence in using the drug remains low. Because the drug is inexpensive and not patent-protected, there are few, if any, incentives 
for drug companies to market $\mathrm{MgSO}_{4}$. Langer et al. ${ }^{14}$ also recognize that adequately packaged $\mathrm{MgSO}_{4}$ is not widely available. An easily administered, inexpensive, safe, and oral alternative to intravenous $\mathrm{MgSO}_{4}$ would be welcome in the developing world, but is not yet available.

In contrast, blood pressure control using intravenous medications was the rule in this report, ${ }^{1}$ and the authors appropriately described their efforts to reduce systolic blood pressure. Although hydralazine is no longer the drug of choice, and may less safe than calcium channel blockers, ${ }^{15}$ the continued use of hydralazine probably reflects availability of this agent, rather than a therapeutic choice. Monitoring blood pressure, and maintaining systolic pressure $<160 \mathrm{mmHg}$, has been shown to be effective in reducing complications secondary to preeclampsia, ${ }^{16}$ and this strategy is one of the key recommendations of the latest issue of the Confidential Enquiry into maternal deaths in the $\mathrm{UK}{ }^{2}$

The authors should be commended for reporting their experience from Côte d'Ivoire, relating to maternal mortality secondary to eclampsia, and for analyzing the associated risk factors in that setting. Brouh et al. ${ }^{1}$ have identified both medical and health systems barriers to reducing maternal mortality from eclampsia. Identification of these barriers is the primary step to begin reducing maternal death rates in their country.

\section{Mortalité maternelle} due à l'éclampsie dans les pays en voie de développement : du progrès mais encore un long chemin à parcourir

Les lecteurs du Journal seront probablement surpris par le contenu de l'article de Brouh et coll. ${ }^{1}$ dans ce numéro, qui décrit la mortalité maternelle et les séjours en réanimation des femmes enceintes en Afrique de l'Ouest. La plupart des lecteurs sont probablement plus familiers avec les rapports sur la mortalité maternelle issus de pays tels que le Royaume-Uni, ${ }^{2}$ la
France $^{3}$ ou les USA. ${ }^{4}$ Ces rapports décrivent généralement un petit nombre de cas, souvent regroupés dans des rapports triennaux pour satisfaire aux exigences méthodologiques des analyses épidémiologiques. Il est intéressant de noter que le rapport triennal britannique $^{2}$ qui en est maintenant à sa septième publication consécutive souligne l'évolution remarquable dans l'épidémiologie des morts maternelles dans ce pays : les facteurs associés à la mort maternelle sont en plein changement et on voit maintenant apparaitre un rôle prédominant de l'obésité, du diabète, du tabagisme et des pathologies psychiatriques. Ces causes seront probablement retrouvées à des degrés divers et dans une échelle de temps variable dans l'ensemble des autres pays occidentaux. Ces facteurs de risque, jusqu'ici presque inconnus dans cette tranche d'âge, prennent aujourd'hui un rôle essentiel. Le nombre croissant des décès secondaires à des causes obstétricales indirectes (aggravation par la grossesse d'une pathologie préexistante) contraste avec la réduction du nombre de décès obstétricaux directs (pathologies causées directement par la grossesse telles que l'hémorragie, la pré-éclampsie ou les complications obstétricales du travail). L'anesthésie est également une cause de mortalité maternelle mais une amélioration considérable peut être observée puisqu'un décès par an lié directement à l'anesthésie est aujourd'hui enregistré dans des pays tels que la France ou le RoyaumeUni. ${ }^{2,3}$ Une amélioration dans l'organisation des soins obstétricaux, le développement de stratégies centrées sur la prévention des causes principales de décès et la formation médicale continue ont été des éléments essentiels de la réduction du taux de décès secondaires aux causes obstétricales directes. Cette évolution positive a été rendue possible en partie parce que le produit national brut de ces pays industrialisés est élevé et que la proportion des finances publiques attribuées à l'amélioration de la santé de la population a été suffisamment importante pour faciliter les changements dans l'organisation des soins. Les limites de ces améliorations semblent avoir été aujourd'hui atteintes puisque les décès de femmes enceintes dans les pays développés touchent maintenant surtout les femmes issues de l'immigration et/ou socialement vulnérables, pour lesquelles les barrières à l'accès aux soins restent importantes. ${ }^{5}$ De façon surprenante, les causes de décès pour cette catégorie de femmes défavorisées sont différentes de celles observées habituellement dans les pays développés et paradoxalement les soignants découvrent en obstétrique les problèmes de santé des classes de niveau socio-économique faible qui représentent la partie inachevée du progrès observé dans nos sociétés industrialisées. 
Il y a cependant de bonnes nouvelles. Le taux de mortalité évitable diminue dans les pays industrialisés et il a un potentiel d'amélioration dans le reste du monde. L'incidence de mortalité maternelle dans les pays développés est de 50 à 100 fois plus faible que ce qui est observé aujourd'hui en Afrique sub-saharienne ou en Asie du Sud-est par exemple. ${ }^{6}$ Dans ces régions, les causes de mortalité maternelle restent celles qui surviennent au cours du travail ou en post-partum immédiat alors que ces situations sont globalement bien contrôlées dans les pays industrialisés. En raison d'une insuffisance de ressources médicales et économiques, les causes directes continuent de contribuer de façon prépondérante à la mortalité maternelle. L'hémorragie obstétricale cause $30 \%$ des morts maternelles et les conséquences de l'hypertension sont responsables de $15 \%$ supplémentaires. ${ }^{7}$ Lorsque l'on ajoute les infections, ces trois facteurs contribuent pour plus de $50 \%$ aux causes de décès maternels en Afrique subsaharienne. ${ }^{6}$ La prise en charge précoce des pathologies obstétricales et notamment la détection précoce de la pré-éclampsie présente une importance considérable. On a ainsi vu se développer des initiatives très intéressantes qui permettent le déploiement de sages-femmes ou d'assistantes obstétricales dans des milieux isolés ou des petits villages pour détecter les complications à un stade précoce. ${ }^{8}$ Dans le cadre de la pré-éclampsie par exemple, la détection précoce de l'hypertension par des mesures répétées de la pression artérielle ou de la protéinurie par l'emploi des bandelettes urinaires est un moyen simple et efficace qui peut prévenir les complications à un stade tardif.

Les morts maternelles surviennent plus souvent à l'hôpital, ${ }^{6}$ suggérant qu'un nombre croissant de femmes enceintes accèdent à des soins organisés et ne sont plus soumises uniquement aux soins fournis par la médecine traditionnelle locale. Une évolution supplémentaire observée dans certains pays est la possibilité d'admission des femmes enceintes en réanimation. Ce niveau de soins est ainsi disponible à Abidjan, capitale de la Côte d'Ivoire ainsi que le rapportent Brouh et ses collaborateurs. ${ }^{1}$ Bien que la Côte d'Ivoire fasse partie de l'Afrique sub-saharienne, elle se situe géographiquement dans sa partie occidentale et présente une couverture sanitaire un peu meilleure que celle des pays voisins. Coccody et Yopougon sont cependant deux des trois seuls hôpitaux universitaires du pays, nous remémorant que l'accès global aux soins très limité dans ces pays et en particulier en Côte d'Ivoire où la mortalité maternelle reste parmi les plus élevées du monde (> 600 pour 100000 naissances vivantes). ${ }^{6}$

Les résultats présentés dans l'étude de Brouh et coll. ${ }^{1}$ peuvent tout de même nous laisser avec un sen- timent mitigé. D'un côté, certains aspects des soins et du fonctionnement de cette réanimation ressemblent beaucoup à nos structures occidentales et l'on peut y observer un taux élevé de césariennes, des patients traités avec de la ventilation artificielle ou encore la disponibilité d'héparines de bas poids moléculaire. Par ailleurs cependant, le nombre de crises d'éclampsie dans ces deux hôpitaux est beaucoup plus élevé que ce que la plupart des anesthésiologistes pourraient en observer au cours de toute leur carrière. De plus, le nombre rapporté ici sous-estime probablement largement la réalité. On sait en effet que la majorité des patientes éclamptiques ne sont pas admises dans ces réanimations en raison du nombre limité de places. ${ }^{9}$ De plus, la disponibilité de médicaments et de matériel modernes dans ces deux unités ne doit pas nous faire oublier le dénuement général qui existe ailleurs dans ces pays. Un audit a ainsi montré qu'en Ouganda, 6 \% seulement des anesthésiologistes avaient les moyens de délivrer une anesthésie sécuritaire pour césarienne. ${ }^{10}$

La mortalité maternelle après éclampsie est élevée dans ces deux structures. En effet, dans la plupart des autres études, la mortalité maternelle secondaire à l'éclampsie est bien plus faible que le taux de $16 \%$ décrit ici. ${ }^{1}$ Dans l'enquête collaborative sur l'éclampsie qui a étudié l'efficacité du sulfate de magnésium $\left(\mathrm{MgSO}_{4}\right)$ en prévention secondaire (c'est-à-dire chez des patientes ayant déjà convulsé), la mortalité maternelle était de 5,1 \% dans les groupes traités par phénytoïne ou diazépam. ${ }^{11}$ Parmi les patientes qui avaient reçu du $\mathrm{MgSO}_{4}$, la mortalité, bien qu'inférieure de $26 \%$ (soit un taux de $3,8 \%$ ), n'était pas significativement différente. Dans une autre étude, réalisée en Inde cette fois sous la forme d'un audit comparant deux périodes - avant puis après introduction du $\mathrm{MgSO}_{4}$ - la mortalité maternelle dans le groupe de femmes ayant reçu du $\mathrm{MgSO}_{4}$ était significativement moindre avec un taux de $4 \%{ }^{12}$ Les décès étaient secondaires à des hémorragies intracrâniennes, des états de mal convulsifs ou des complications associées telles que l'insuffisance rénale, l'ictère, l'inhalation de liquide gastrique ou encore l'œdème pulmonaire. Dans les études au cours desquelles le $\mathrm{MgSO}_{4}$ était administré à des pré-éclamptiques sévères mais qui n'avaient pas encore convulsé (prévention primaire), la mortalité maternelle était encore plus faible $(0,4 \%$ par exemple dans l'étude Magpie). ${ }^{13}$ En d'autres termes, la sévérité de la maladie sous-jacente et l'absence d'emploi du $\mathrm{MgSO}_{4}$ sont des facteurs majeurs d'augmentation du risque de décès. Ainsi que le décrivent Brouh et coll., ${ }^{1}$ le $\mathrm{MgSO}_{4}$ n'était pas utilisé dans leur unité au moment de l'étude et le diazépam, bien que moins efficace en prévention de la survenue de convulsions, restait le 
médicament de référence. Cette situation n'est pas unique. Des barrières à l'emploi du $\mathrm{MgSO}_{4}$ existent dans de nombreux pays et les facteurs facilitant l'accès au produit ont été récemment décrites. ${ }^{14}$ La plupart des pays à faibles ressources économiques n'ont pas de protocole exigeant l'emploi du $\mathrm{MgSO}_{4}$, il existe des limitations financières ou logistiques et de nombreux médecins perçoivent le produit comme dangereux et difficile d'emploi. De nombreuses structures dans les pays en voie de développement n'ont pas l'équipement nécessaire et la formation pour administrer le produit et de nombreuses patientes avec éclampsie imminente ne reçoivent pas le $\mathrm{MgSO}_{4}$ avant la première convulsion. La mortalité et la morbidité induites par les erreurs d'administration ou la toxicité du $\mathrm{MgSO}_{4}$ ont été largement diffusées et la plupart des soignants n'ont pas été formés à son utilisation. Du fait de son faible prix et de son ancienneté, il existe peu d'intérêt de la part des compagnies pharmaceutiques pour favoriser le développement du produit. Langer et coll. ${ }^{14}$ ont aussi montré que la présentation souvent inadaptée du $\mathrm{MgSO}_{4}$ (volume inadapté des poches de perfusion) ne facilite pas son emploi. Une alternative peu coûteuse, sans toxicité et administrable par voie orale serait vraiment la bienvenue dans les pays en voie de développement mais n'est pas pour l'instant disponible.

En contrepoint, l'étude décrit une prise en charge efficace de la pression artérielle. Bien que l'hydralazine ne soit plus le médicament de choix et présente plus d'effets indésirables que les inhibiteurs calciques, ${ }^{15}$ l'emploi de ce produit traduit plus probablement sa disponibilité plutôt qu'un réel choix thérapeutique. La surveillance de la pression artérielle et le maintien d'une pression systolique inférieure à $160 \mathrm{mmHg}$ sont des moyens efficaces pour réduire le risque de complications secondaires à la pré-éclampsie ${ }^{16}$ et cette stratégie est l'un de points essentiels sur lequel insiste le dernier rapport britannique sur la mortalité maternelle. ${ }^{2}$

Les auteurs de ce travail doivent être félicités pour avoir rapporté leur expérience concernant la mortalité maternelle secondaire à l'éclampsie en Côte d'Ivoire et pour avoir analysé les facteurs favorisants. Brouh et coll. ${ }^{1}$ ont ainsi identifié des barrières médicales et plus largement des défauts du système de soins qui contribuent au taux élevé de mortalité maternelle. L'identification de ces barrières et insuffisances est le premier pas vers une réduction de la mortalité maternelle en Côte d'Ivoire.

\section{References}

1 Broub $\Upsilon$, Ndjeundo PG, Tetchi YD, Amonkou AA, Pete $\Upsilon$, rapobi $\Upsilon$. Les éclampsies en centre hospitalier en
Côte d'Ivoire: prise en charge, évolution et facteurs pronostiques. Can J Anesth 2008; 55: 423-8.

2 CEMACH Publications. Saving mothers'lives: reviewing maternal deaths to make motherhood safer - 2003-2005. Confidential Enquiry into Maternal and Child Death. December 2007. The seventh report of the confidential enquiries into maternal deaths in the United Kingdom. Available from URL; http://www.cemach.org.uk/Publications/CEMACHPublications/Maternal-and-Perinatal-Health.aspx (accessed April 2008).

3 Comité National d'experts sur la mortalité maternelle. La mortalité maternelle en France: considérations épdiémiologiques et cliniques (1999-2001) et recommandations. Bulletin Epidémiologique Hebdomadaire thématique 50, 12 décembre 2006: 395-399. Available from URL; www.sante.gouv.fr/htm/pointsur/maternite/rapport3.htm (accessed April 2008).

4 Minino AM, Heron MP, Murphy SL, Kochanek KD; Centers for Disease Control and Prevention National Center for Health Statistics National Vital Statistics System. Deaths: final data for 2004. Natl Vital Stat Rep 2007; 55: 1-119.

5 Bragg R. Maternal deaths and vulnerable migrants. Lancet 2008; 371: 879-81.

6 Ronsmans C, Graham WJ; Lancet Maternal Survival Series Steering Group. Maternal mortality: who, when, where, and why. Lancet 2006; 368: 1189-200.

7 Khan KS, Wojdyla D, Say L, Gulmezoglu AM, Van Look PF. WHO analysis of causes of maternal death: a systematic review. Lancet 2006; 367: 1066-74.

8 Shankar A, Sebayang S, Guarenti L, Utomo B, Islam M, Fauveau V, Jalal $F$. The village-based midwife programme in Indonesia. Lancet 2008; 371: 1226-9.

9 Sombie I, Meda N, Hounton S, Bambara M, Ouedraogo $T W$, Graham W. Missing maternal deaths: lessons from Souro Sanou University Hospital in Bobo-Dioulasso, Burkina Faso. Trop Doct 2007; 37: 96-8.

10 Hodges SC, Mijumbi C, Okello M, McCormick BA, Walker IA, Wilson IH. Anaesthesia services in developing countries: defining the problems. Anaesthesia 2007; 62: 4-11.

11 Anonymous. Which anticonvulsant for women with eclampsia? Evidence from the Collaborative Eclampsia Trial. Lancet 1995; 345: 1455-63. Erratum in: Lancet 1995; 346: 258.

12 Sawhney H, Aggarwal N, Biswas R, Vasishta K, Gopalan $S$. Maternal mortality associated with eclampsia and severe preeclampsia of pregnancy. J Obstet Gynaecol Res 2000; 26: 351-6.

13 Altman D, Carroli G, Duley L, et al.; Magpie Trial Collaborative Group. Do women with pre-eclampsia, and their babies, benefit from magnesium sulphate? 
The Magpie Trial: a randomised placebo-controlled trial. Lancet 2002; 359: 1877-90.

14 Langer A, Villar J, Tell K, Kim T, Kennedy S.

Reducing eclampsia-related deaths - a call to action.

Lancet 2008; 371: 705-6.

15 Magee LA, Cham C, Waterman EJ, Ohlsson A,

von Dadelszen $P$. Hydralazine for treatment of severe hypertension in pregnancy: meta-analysis. BMJ 2003; 327: 955-60.

16 Martin JN Jr, Thigpen BD, Moore RC, Rose CH, Cushman J, May W. Stroke and severe preeclampsia and eclampsia: a paradigm shift focusing on systolic blood pressure. Obstet Gynecol 2005; 105: 246-54.

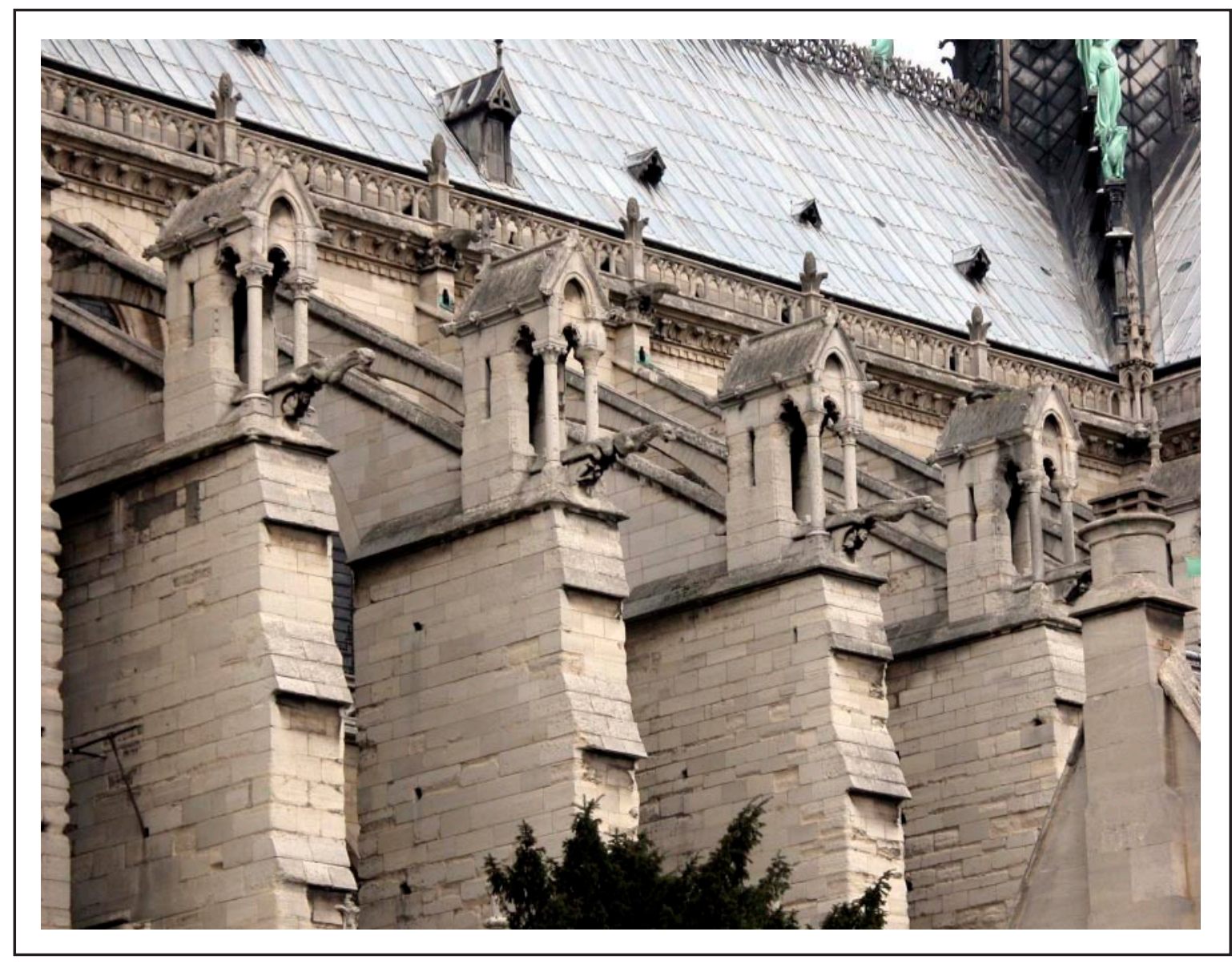

Flying Buttresses - Notre Dame, Paris 